Gastric varices were classified as: GOV1 4 (31\%), GOV2 6 (46\%), IGV1 3(31\%). There were co-existing oesophageal varices in 9 of 13 $(69 \%)$, of which 3 were banded in addition but not actively bleeding. Mean thrombin dose used was 1125IU (range 500-2000). Immediate haemostasis was achieved in all 15 cases. Propanolol was commenced post endoscopy in 14 (93\%) patients and maintained at a mean dose of 80mg/day (SD 35, range 20-160).

Median follow up time was 129 days (range 9-753). No patient received TIPSS or liver transplantation. Rebleeding occurred in 3 (20\%) patients, at 14, 43 and 299 days respectively. All 3 patients died following rebleeding ( 2 declined treatment, 1 pre-hospital arrest). There were 7 deaths in total during the study period, the remainder due to liver failure (2), pneumonia (1), metastatic cancer (1). Cumulative survival at 1, 3, 6, 12 months was $73 \%, 59 \%, 59 \%$, and $50 \%$ respectively.

Conclusion Single dose thrombin injection in our series appears to be a safe, easily administered and effective endoscopic therapy for acutely bleeding oesophagogastric varices. Mortality however remains high due to their underlying liver disease.

Disclosure of Interest None Declared.

\section{PWE-060 THE HOSPITAL ANXIETY AND DEPRESSION SCALE (HADS) PREDICTS PAIN AND DISTRESS AT ENDOSCOPY}

doi:10.1136/gutjnl-2013-304907.349

'A J Irvine, 1,"M Kurien, 'R Harrold, 'L Taylor, 'D S Sanders. 'Department of Gastroenterology, Royal Hallamshire Hospital, Sheffield, UK

Introduction Tolerability of endoscopy is variable, with pain and distress influencing overall experience. Currently, there is a paucity of work evaluating distress, with no reliable tools established as predictors of endoscopic tolerability. A recent study found higher levels of discomfort during colonoscopy in patients scoring 11 or more (out of a maximum of 21) on the anxiety portion of the HADS questionnaire evaluated post-procedure. ${ }^{1}$ Our study evaluates the pre-endoscopic use of HADS and its value in predicting procedural pain and distress. Methods Consecutive patients attending for clinically indicated OGD or colonoscopy were prospectively recruited between September 2011 and June 2012 at a University hospital. Prior to endoscopy, patients completed the HADS questionnaire and were familiarised with the 10-point numeric rating scale used to assess expected pain and distress and post-procedural pain and distress. Patients with high HADS anxiety scores (HADS $\geq 11$ ) were then compared with those with low scores (HADS $\leq 10)$, with the cut off value of 11 defined in accordance with the original HADS paper. ${ }^{2}$ Data was analysed using SPSS version 20, with a Mann Whitney U test used to determine differences between procedural pain and distress scores. Results 610 patients were prospectively recruited (280 male patients, median age 56 years, range 17-90 years, 306 OGD's), with $21 \%(128 / 610)$ having HADS anxiety scores $>11$. Of these individuals, 51\% (65/128) had elevated procedural pain, with 53\% (68/128) having elevated procedural distress. By comparison in patients with HADS anxiety scores < 10, only $32 \%(154 / 482)$ had elevated procedural pain and $37 \%(176 / 482)$ had elevated distress. Comparisons between the two groups (HADS $\geq 11$ and those with $\mathrm{HADS} \leq 10$ ) demonstrated significant differences ( $p=0.001$ for pain and $p<0.001$ for distress). Median scores for the two groups are highlighted in Table 1.

\section{Abstract PWE-060 Table 1 Median procedural pain} and distress scores

\begin{tabular}{lll}
\hline & \multicolumn{2}{l}{ HADS anxiety score of 11+ } \\
\cline { 2 - 3 } & Less than 10 & 11 or more \\
\hline Median Procedural pain Score & 3 & 5 \\
Median Procedural distress Score & 2 & 5 \\
\hline
\end{tabular}

Conclusion This is the first study demonstrating how the HADS could be used to predict endoscopic tolerability, with HADS anxiety scores $\geq 11$ associated with over a $50 \%$ chance of having procedural pain and distress. Adopting HADS into pre-endoscopy assessments could help identify patients likely to poorly tolerate endoscopy, leading to earlier consideration of sedation, analgesia and other endoscopic measures to minimise pain and distress.

Disclosure of Interest None Declared.

\section{REFERENCES}

1. Elphick $D$ et al. Factors associated with abdominal discomfort during colonoscopy: a prospective analysis. European Journal of Gastroenterology and Hepatology. 2009 Sep; 21(9):1076-82.

2. Zigmond AS et al. The hospital anxiety and depression scale. Acta Psychiatr Scand. 1983 Jun; 67(6):361-70

\section{PWE-061 ERCP PRACTICE IN A UK DISTRICT HOSPITAL- ARE WE MEETING THE STANDARDS?}

doi:10.1136/gutjnl-2013-304907.350

1."M Kumar, 'C Ruscanu, 'D Hanumantharaya, 'W Melia, 'R Ede. 'Darent Valley Hospital, Dartford, UK

Introduction The 2004 NCEPOD report "Scoping our Practice"1 had been highly critical of certain aspects of ERCP practise in UK, raising specific concerns about case selection and sedation practise. We analysed our own ERCP practise in a medium sized district hospital with a moderate case workload and a growing proportion of elderly population.

Methods Retrospective data was collected from 263 ERCPs performed between 2009-2011. Comprehensive information regarding demographics, indications, success and complication rates was recorded from ERCP reports and case notes and our practise was compared to NCEPOD recommendations.

Results 263 (n) ERCPs were included in this study. Median age was 72 (range $=16-98$ ), 63\% were females. $55 \%$ of patients were ASA grade 3-4. 84\% of ERCPs were of grade 1 difficulty. All ERCP referrals were reviewed and authorised by a consultant gastroenterologist. Indications for ERCP were choledocholithiasis (63\%), pancreatic or biliary malignancy with obstructive jaundice (18\%), stent removal/replacement (10\%), dilatation of biliary ducts with abnormal liver function tests $(10 \%)$ and others $(4 \%) .>90 \%$ of ERCPs were performed with a therapeutic intent and success was achieved in $86 \%$ of ERCPs at first attempt. Our successful cannulation rate was of $92 \%$. Only $9.1 \%$ of cases were referred to tertiary centres for further management. Prophylactic oral ciprofloxacin was used in $60 \%$ of patients. Patients received a combination of midazolam and pethidine with a mean dose $( \pm \mathrm{SD})$ of $3.2 \mathrm{mg}( \pm 2.03)$ and $44.3 \mathrm{mg}$ $( \pm 16.05)$ respectively. Reversal with flumazenil or naloxone was not required in any of the patients included in this study. Biliary sphincterotomy was performed in $60 \%(156)$, pre-cut sphincterotomy in $2.6 \%(7)$, stricture dilatation in $9.5 \%(25)$, biliary stenting $30.4 \%(80)$, balloon sphincteroplasty $3 \%(8)$, balloon trawl $67 \%(177)$ and mechanical lithotripsy $8.7 \%(23) .78 .7 \%$ of malignant strictures were successfully stented (37). Overall complication rate was $5.7 \%$ - moderate haemorrhage requiring blood transfusion in 1.5\%(4), post ERCP pancreatitis in 2\%(6), sepsis 1.9\%(5), duodenal perforation $0.7 \%(1)$ and respiratory arrest in $0.7 \%(1)$. 30 day mortality rate was $0.76 \%(2)$.

Conclusion In contrast to NCEPOD report, our audit demonstrates that ERCP practise is effective, safe and of high quality in a district general hospital setting. Complication and mortality rates are minimal and comparable to national standards, even in the elderly population. Post ERCP very low sepsis rate is most likely due to use of prophylactic antibiotics (Ciprofloxacin).

Disclosure of Interest None Declared. 